\title{
Impact of apoE genotype on postprandial lipid and glucose responses in healthy men
}

\author{
V. Calabuig-Navarro ${ }^{1}$, C. M. Walden ${ }^{2}$, J. A. Lovegrove ${ }^{1}$, A. M. Minhane ${ }^{1}$ and K. G. Jackson ${ }^{1}$ \\ ${ }^{1}$ Department of Food and Nutritional Sciences, University of Reading, RG6 6AP, UK and ${ }^{2}$ Unilever Discover, Colworth \\ Science Park, Sharnbrook, MK44 ILQ, UK
}

ApoE, a protein found associated with TAG-rich lipoproteins in the bloodstream, plays an important role in fat transport and its clearance from the circulation. ApoE is present in three isoforms (apoE2, E3 and E4) and it is well recognised that apoE4 carriers (25\% of the population) have a 40-50\% higher risk of CVD. The apoE genotype is thought to play a role in an individual's responsiveness to dietary fat change with the greatest reduction in LDL cholesterol levels evident in apoE4 carriers following the switch to a low-fat/ low-cholesterol diet. However, the responsiveness to acute changes in dietary fat composition is relatively unknown.

Our aim was to examine the impact of the apoE genotype to meals of varying fatty acid composition on postprandial plasma TAG, glucose and NEFA responses. Twenty-one healthy men (mean (SD), age 48 (16) years and BMI 26 (3) $\mathrm{kg} / \mathrm{m}^{2}$ ), recruited according to apoE genotype $(10$ apoE3/3, 11 apoE3/E4), received in random order a mixed meal containing $50 \mathrm{~g}$ of fat rich in either SFA, unsaturated fatty acids (UNSAT) or SFA with fish oils (FO, $5 \mathrm{~g}$ DHA and $0.6 \mathrm{~g}$ EPA) on three separate occasions. Fasting and regular postprandial blood samples were collected until 480 min after the meal. Plasma TAG, NEFA and glucose were measured using automated assays.

\begin{tabular}{|c|c|c|c|c|c|c|c|c|c|c|c|c|}
\hline & \multicolumn{4}{|c|}{$\mathrm{TAG}^{\dagger}$} & \multicolumn{4}{|c|}{ Glucose $\dagger$} & \multicolumn{4}{|c|}{$\mathrm{NEFA} \dagger$} \\
\hline & \multicolumn{2}{|c|}{ Fasting } & \multicolumn{2}{|c|}{ AUC } & \multicolumn{2}{|c|}{ Fasting } & \multicolumn{2}{|c|}{ AUC } & \multicolumn{2}{|c|}{ Fasting } & \multicolumn{2}{|c|}{ AUC } \\
\hline & Mean & $\overline{\mathrm{SE}}$ & Mean & $\mathrm{SE}$ & Mean & $\overline{\mathrm{SE}}$ & Mean & $\mathrm{SE}$ & $\overline{\text { Mean }}$ & $\mathrm{SE}$ & $\overline{\text { Mean }}$ & SE \\
\hline \multicolumn{13}{|c|}{$\mathrm{E} 3 / \mathrm{E} 3(n$ 10) } \\
\hline SFA & 1.3 & 0.2 & 926.7 & 144.5 & 5.66 & 0.1 & 2737 & 55.6 & 0.45 & 0.04 & 182.7 & 11.1 \\
\hline $\mathrm{FO}$ & 1.18 & 0.1 & 823.9 & 101.4 & 5.88 & 0.1 & 2732.6 & 82.6 & 0.4 & 0.05 & 163.1 & 9 \\
\hline UNSAT & 1.47 & 0.3 & 1002.5 & 169.7 & 5.71 & 0.1 & 2674.2 & 68.9 & 0.42 & 0.05 & 166.6 & 11.1 \\
\hline \multicolumn{13}{|c|}{$\mathrm{E} 3 / \mathrm{E} 4\left(\begin{array}{ll}n & 11\end{array}\right)$} \\
\hline SFA & 1.59 & 0.2 & 996.1 & 105.3 & 5.57 & 0.1 & 2795.8 & 61.8 & 0.38 & 0.07 & 163.6 & 14.7 \\
\hline FO & 1.46 & 0.1 & 897.8 & 111.6 & 5.4 & 0.1 & 2707.7 & 79.9 & 0.44 & 0.04 & 163.1 & 8.7 \\
\hline UNSAT & 1.6 & 0.2 & 986.5 & 106.9 & 5.57 & 0.1 & 2729 & 64.3 & 0.44 & 0.05 & 166.6 & 12.6 \\
\hline
\end{tabular}

${ }^{\dagger}$ Units for fasting concentration are $\mathrm{mmol} / \mathrm{l}$ and $\mathrm{AUC}$ are $\mathrm{mmol} / \mathrm{l} \times 480 \mathrm{~min}$.

There was no significant impact of apoE genotype on fasting or area under the curve (AUC) for the postprandial TAG, glucose and NEFA responses after the three meals. For the genotype groups combined, the time to reach peak TAG concentration was shown to be later following the SFA compared with the UNSAT meal $(P=0.004)$. Peak glucose and NEFA concentrations were also different after the meals, with higher concentrations reached after the SFA compared with the UNSAT meal $(P<0.02)$.

In the group as a whole, a difference in the time to reach peak TAG, and peak glucose and NEFA concentrations were observed between the meals rich in SFA and unsaturated fatty acids. However, our data indicate that postprandial lipid and glucose responses were not different between our healthy male apoE3/E3 or apoE4 carriers suggesting that apoE genotype did not affect the acute postprandial responsiveness to manipulating meal dietary fatty acid composition. 